\title{
Body Composition and Metabolic Improvement in Patients Followed Up by a Multidisciplinary Team for Obesity in China
}

\author{
Difei Lu $\mathbb{D}^{1},{ }^{1}$ Zhenfang Yuan $\left(\mathbb{D},{ }^{1}\right.$ Lihua Yang, ${ }^{2}$ Yong Jiang, ${ }^{3}$ Min Li, $^{4}$ Yuanzheng Wang, ${ }^{5}$ \\ Lulu Jing, ${ }^{2}$ Rongli Wang, ${ }^{4}$ Junqing Zhang $\left({ }^{1},{ }^{1}\right.$ and Xiaohui Guo ${ }^{1}{ }^{1}$ \\ ${ }^{1}$ Department of Endocrinology, Peking University First Hospital, China \\ ${ }^{2}$ Department of Clinical Nutrition, Peking University First Hospital, China \\ ${ }^{3}$ Department of General Surgery, Peking University First Hospital, China \\ ${ }^{4}$ Department of Rehabilitation, Peking University First Hospital, China \\ ${ }^{5}$ Department of Integrative Traditional Chinese Medicine and Western Medicine, Peking University First Hospital, China
}

Correspondence should be addressed to Zhenfang Yuan; zhenfang_yuan@sina.cn

Received 18 September 2020; Revised 1 October 2020; Accepted 11 March 2021; Published 29 July 2021

Academic Editor: Carlo Sasso

Copyright $(2021$ Difei Lu et al. This is an open access article distributed under the Creative Commons Attribution License, which permits unrestricted use, distribution, and reproduction in any medium, provided the original work is properly cited.

\begin{abstract}
Background. This study evaluated the effectiveness of the multidisciplinary team (including a specialist, a dietitian, a physical exercise trainer, a surgeon for bariatric surgery, an acupuncturist, and several health educators) for obesity management and the body composition change and improvements in metabolic biomarkers during a 2-year follow-up. Materials and Methods. A total of 119 patients participated in the multidisciplinary team for obesity. Patients were followed up at 3 months, 6 months, 1 year, 18 months, and 2 years after their first visit. Individuals were divided into the high-protein diet (HPD) and standardprotein diet (SPD) group according to their results on a diet questionnaire that they filled out during follow-up. Results. After 1.2 years, the mean body weight of the participants dropped from $89.7 \mathrm{~kg}$ to $80.9 \mathrm{~kg}(p<0.001)$. The body adiposity index was reduced from 33.9 to $32.0(p<0.001)$, while the fat-free mass index from 17.0 to $15.2(p=0.043)$. Fasting glucose and HbA1c were also lower after treatment $(p=0.002$ and 0.038 for FPG and HbAlc, respectively). Fasting insulin and HOMA-IR were reduced $(p=0.002$ and $<0.001$ for fasting insulin and HOMA-IR, respectively). HDL-c increased along with weight loss $(1.06 \mathrm{mmol} / \mathrm{L}$ vs. $1.19 \mathrm{mmol} / \mathrm{L}, p<0.001)$, and transaminase levels significantly dropped $(p=0.001$ and 0.021 for ALT and AST, respectively). During treatment, mean protein intake was $29.9 \%$ in the HPD group and $19.5 \%$ in the SPD group ( $p<0.001)$. Weight loss, reduction of visceral fat area, maintenance of lean body mass, body adiposity index, and fat-free mass index showed no statistical significance between the HPD and SPD groups, as well as glucose metabolic variables. Conclusions. A multidisciplinary team for obesity management could significantly reduce body weight and improve metabolic indicators, including HDL-c, transaminase, and insulin resistance. A high-protein diet does not produce better weight control or body composition compared with a standard calorie-restricted diet.
\end{abstract}

\section{Introduction}

The prevalence of obesity has been gradually increasing over the past 30 years. According to the most recent global estimates, over 500 million individuals are obese, which is creating a heavy health burden on patients and the social economy [1]. The most common complications of obesity include type 2 diabetes, hypertension, and hyperlipidemia. Regular physical activity, healthy diets, weight-loss medicines, and bariat- ric surgery are common treatments for obesity [2]. Moreover, a recent study proved that acupuncture might be effective against obesity [3]. Besides lifestyle changes, behavior intervention, such as patient education, motivating to a straightforward goal setting, and self-monitoring, is also of great importance [4]. Therefore, a multidisciplinary team that comprises physicians, dietitians, health educators, and physical activity trainers is recommended to execute lifestyle intervention for obesity patients in a more efficient way [5]. A 
consecutive follow-up by a multidisciplinary team can modulate an individualized lifestyle intervention, based on clinical evidence [6-8].

Some randomized controlled trials showed that a protein-rich hypocaloric diet is effective in reducing weight [9]. Other studies suggest that high-protein intake could increase satiety and reduce calorie intake. The proportion of macronutrients that a patient should take in order to reduce weight depends on individual goals, activity level, age, health, genetics, and much more.

The aim of this study was to evaluate the effectiveness of the multidisciplinary team for obesity management and investigate the body composition change and the improvements of metabolic biomarkers during a 2-year follow-up between a high-protein diet and a standard low-calorie diet. Our multidisciplinary outpatient clinic for obesity patients, which was launched on June 1, 2015, in Peking University First Hospital (PKUFH), includes an endocrinology specialist, a dietitian, a physical exercise trainer, a surgeon for bariatric surgery, an acupuncturist, and several health educators.

\section{Materials and Methods}

2.1. Study Design. Male and female individuals aged 18-75 years old with BMI $>24 \mathrm{~kg} / \mathrm{m}^{2}$ defined as overweight and those $>28 \mathrm{~kg} / \mathrm{m}^{2}$ defined as obese were enrolled in the MDT clinic. All participants had given written informed consent at the first visit. Ethical approval was given from the Ethics Committee of Peking University First Hospital.

Diseases of secondary obesity, including Cushing's disease and hypothyroidism as exclusion criteria, were ruled out at the first visit. A series of educational programs were provided to the patients at the first visit to explain the goal of the study and the purpose of the treatment. During their first visit, participants' body height $(\mathrm{BH})$, body weight (BW), waist circumference (WC), and hip circumference (HC) were measured and recorded by health educators. Demographic data, including age, gender, smoking status, daily food intake, and past medical history (type 2 diabetes, hypertension, hyperlipidemia, hyperuricemia, and nonalcoholic fatty liver disease (NAFLD)), were analyzed through a questionnaire.

After the anthropometric evaluations, a fasting venous blood sample was withdrawn to obtain baseline data for a full-scale evaluation of weight-related comorbidities, including liver function, lipid profile, HbAlc, fasting plasma glucose, plasma uric acid, and fasting plasma insulin.

Body fat percentage (BF\%), lean body mass (kg), muscle mass of lower extremities $(\mathrm{kg})$, and visceral fat area $\left(\mathrm{cm}^{2}\right)$ along with other body composition variables were determined using bioelectrical impedance analysis (InBody ${ }^{\circledR} 720$ Medical Body Composition Analyzer, Biospace Co., Ltd., Korea). Body adiposity index (BAI) was calculated from height and hip circumference $(\mathrm{HC})$ as follows: $\mathrm{BAI}=\left(\mathrm{HC}(\mathrm{cm}) /\right.$ height $\left.(\mathrm{m})^{1.5}\right)$ -18 [10]. The fat-free mass index (FFMI) was calculated in accordance with dividing the lean mass $(\mathrm{kg})$ by height squared (m) [11]. The homeostasis model assessment for insulin resistance (HOMA-IR) was calculated from fasting plasma glucose (FPG, $\mathrm{mmol} / \mathrm{L})$ and fasting insulin $(\mathrm{mU} / \mathrm{mL})$ using the follow- ing equation: $\mathrm{HOMA}-\mathrm{IR}=$ fasting insulin $\times$ FPG/22.5 [12]. HOMA- $\beta$ for $\beta$-cell function was determined with the equation HOMA $-\beta=(20 \times$ fasting insulin $) /(\mathrm{FPG}-3.5)[12]$.

2.2. Diets and Intervention. At each visit, patients met with the whole multidisciplinary team, which consisted of a physician, a dietitian, an exercise trainer, a surgeon for bariatric surgery, an acupuncturist, and several health educators. An individualized meal plan and an exercise guide were provided at the initial visit, and pharmacotherapy or acupuncture procedure was recommended. If no significant weight reduction was observed three to six months after standard management, bariatric surgery was recommended.

Regular follow-up was scheduled at 3 months (V2), 6 months (V3), 1 year (V4), 18 months (V5), and 2 years (V6) after the initial visit (V1). Body weight and recent food intake were recorded at each visit by the nursing staff. Patients were enrolled from June 2015 to June 2017, and they were regularly followed up by the multidisciplinary team. Individualized management was modulated at every visit for each patient. A high-protein diet was defined as daily protein intake above $20 \%$ of total calorie intake, in the meantime meeting the criteria of exceeding $1.5 \mathrm{~g} / \mathrm{kg}$ body weight/day. Patients were allocated to a standard protein diet (SPD) group and a high-protein diet (HPD) group based on their food intake proportion during follow-ups.

2.3. Statistical Analysis. SPSS version 18.0 (IBM, USA) was used for analyses. Continuous variables were described as mean \pm standard deviation (SD) and categorical variables as a percentage. A paired $t$-test was used to compare withingroup mean differences between visits. A chi-square test for independence was used to compare the differences between categorical variables. Student's $t$-test was used for means of metabolic indicators between HPD and SPD groups.

\section{Results}

3.1. Baseline Characteristics. A total of 119 patients received integrated care and follow-up until June 2017. Among them, $32(26.9 \%)$ patients were prescribed metformin, $16(13.4 \%)$ patients received acupuncture therapy, and $3(2.7 \%)$ patients received glucagon-like peptide 1 (GLP-1), an agonist. There was no significant difference in baseline characteristics and anthropometric data between high-protein diet (HPD) and standard-protein diet (SPD) groups at their first visit (Table 1). The majority of the patients were female $(72.3 \%)$ and young (aged from 26 to 52 years old). The mean body weight at the initial visit was $89.7 \pm 20.1 \mathrm{~kg}$, and the mean body mass index (BMI) was $32.7 \mathrm{~kg} / \mathrm{m}^{2}$. Their mean followup duration was 1.2 years. There were high comorbidity rates of metabolic diseases. Among the patients, 38.8\% suffered hyperlipidemia, $39.5 \%$ had type 2 diabetes, $37.4 \%$ had hypertension, $60.4 \%$ had nonalcoholic fatty liver disease (NAFLD), $21.4 \%$ had hyperuricemia, and $38.7 \%$ had metabolic syndrome.

3.2. Improvements in Body Weight and Metabolic Indicators. After a 1.2-year mean duration of multidisciplinary treatment in PKUFH, the mean body weight of the participants 
TABLE 1: Comparison of the demographic, anthropometric, and biochemical variables between SPD and HPD groups.

\begin{tabular}{|c|c|c|c|}
\hline & HPD group $(n=27)$ & SPD group $(n=92)$ & $p$ value* \\
\hline Age (years) & $36.1 \pm 9.7$ & $40.0 \pm 12.1$ & 0.123 \\
\hline Sex category (male/female) & $6 / 21$ & $29 / 63$ & 0.351 \\
\hline Follow-up duration (years) & $1.1 \pm 0.7$ & $1.2 \pm 0.6$ & 0.339 \\
\hline Height (cm) & $164.7 \pm 8.5$ & $164.9 \pm 8.9$ & 0.924 \\
\hline Weight (kg) & $89.4 \pm 19.9$ & $89.8 \pm 20.5$ & 0.930 \\
\hline BMI $\left(\mathrm{kg} / \mathrm{m}^{2}\right)$ & $32.7 \pm 5.5$ & $32.7 \pm 5.3$ & 0.951 \\
\hline Waist circumference $(\mathrm{cm})$ & $99.6 \pm 16.1$ & $100.5 \pm 12.4$ & 0.765 \\
\hline Hip circumference $(\mathrm{cm})$ & $109.9 \pm 11.7$ & $109.9 \pm 10.8$ & 0.989 \\
\hline Body fat (\%) & $41.0 \pm 6.9$ & $41.1 \pm 5.5$ & 0.889 \\
\hline Systolic blood pressure (mmHg) & $126.8 \pm 15.5$ & $127.9 \pm 16.3$ & 0.766 \\
\hline Diastolic blood pressure (mmHg) & $81.1 \pm 6.7$ & $82.4 \pm 10.3$ & 0.530 \\
\hline History of T2DM $(n, \%)$ & $10,37.0 \%$ & $37,40.2 \%$ & 0.766 \\
\hline Fasting plasma glucose $(\mathrm{mmol} / \mathrm{L})$ & $6.4 \pm 2.4$ & $6.3 \pm 2.0$ & 0.832 \\
\hline HbAlc (\%) & $6.8 \pm 1.8$ & $6.8 \pm 1.7$ & 0.974 \\
\hline Fasting insulin $(\mu \mathrm{IU} / \mathrm{mL})$ & $16.6 \pm 9.5$ & $19.6 \pm 10.5$ & 0.251 \\
\hline Fasting C-peptide (ng/mL) & $2.9 \pm 1.0$ & $3.3 \pm 1.2$ & 0.138 \\
\hline НOMA- $\beta$ & $209.4 \pm 59.1$ & $198.0 \pm 22.0$ & 0.825 \\
\hline HOMA-IR & $4.7 \pm 3.2$ & $5.1 \pm 3.0$ & 0.679 \\
\hline $\operatorname{ALT}(\mathrm{U} / \mathrm{L})$ & $41.9 \pm 33.6$ & $39.2 \pm 33.3$ & 0.744 \\
\hline AST (U/L) & $26.7 \pm 15.7$ & $26.2 \pm 16.2$ & 0.891 \\
\hline Uric acid $(\mu \mathrm{mol} / \mathrm{L})$ & $364.1 \pm 97.6$ & $374.9 \pm 95.7$ & 0.639 \\
\hline Baseline calorie intake (kcal/d) & $1681.9 \pm 329.5$ & $2141.2 \pm 651.6$ & 0.037 \\
\hline Protein intake proportion (\%) & $16.4 \pm 3.2$ & $17.2 \pm 2.6$ & 0.875 \\
\hline Fat intake proportion (\%) & $31.6 \pm 7.0$ & $33.3 \pm 7.5$ & 0.532 \\
\hline Glucose intake proportion (\%) & $52.2 \pm 8.2$ & $52.4 \pm 7.2$ & 0.955 \\
\hline Visceral fat area $\left(\mathrm{cm}^{2}\right)$ & $173.8 \pm 46.0$ & $174.3 \pm 40.2$ & 0.960 \\
\hline Lean body mass (kg) & $52.3 \pm 11.3$ & $52.3 \pm 12.0$ & 0.989 \\
\hline Muscle mass of lower extremities (kg) & $16.3 \pm 3.4$ & $16.2 \pm 4.0$ & 0.851 \\
\hline Body adiposity index & $34.1 \pm 5.1$ & $34.0 \pm 4.8$ & 0.901 \\
\hline Fat-free mass index & $16.7 \pm 6.4$ & $17.5 \pm 5.6$ & 0.538 \\
\hline
\end{tabular}

T2DM: type 2 diabetes mellitus; ALT: glutamic-pyruvic transaminase; AST: glutamic-oxaloacetic transaminase. ${ }^{*} \chi^{2}$ test for categorical variables and Student's $t$ -test for continuous variables.

dropped from $89.7 \mathrm{~kg}$ to $80.9 \mathrm{~kg}(p<0.001)$, accompanied with the loss of lean body mass of $1.2 \mathrm{~kg}(51.7 \mathrm{~kg}$ vs. $50.5 \mathrm{~kg}$, $p<0.001)$ and lower extremity muscle mass of $0.4 \mathrm{~kg}$ (16.1 kg vs. $15.7 \mathrm{~kg}, p<0.001)$. Body adiposity index was significantly reduced from 33.9 to $32.0(p<0.001)$, while fat-free mass index from 17.0 to $15.2(p=0.043)$.

Regarding glucose metabolism, the distinctive reduction of FPG and HbAlc indicated improved glucose control after treatment $(p=0.002$ and 0.038 for FPG and $\mathrm{HbA1c}$, respectively, Table 2). Also, predominance change was discovered in insulin resistance based on the decline of fasting insulin level and HOMA-IR $(p=0.002$ and $<0.001$ for fasting insulin and HOMA-IR, respectively, Table 2). Pancreatic $\beta$-cell function did not display a significant change $(p=0.134$ for HOMA- $\beta$ ).
Concerning lipid metabolism, HDL-c remarkably increased along with weight loss $(1.06 \mathrm{mmol} / \mathrm{L}$ vs. $1.19 \mathrm{mmol} / \mathrm{L}, p<0.001$ ), but triglyceride, TCHO, and LDL$c$ remained unchanged. There was a high comorbidity rate of NAFLD in the participants, and the transaminase levels were significantly reduced after weight management ( $p=0.001$ and 0.021 for ALT and AST, respectively, Table 2).

3.3. Weight Loss in HPD and SPD Groups. During the diet, the mean calorie intake was $1389 \mathrm{kcal} / \mathrm{d}$ in the HPD group with $29.9 \%$ protein proportion and $1393 \mathrm{kcal} / \mathrm{d}$ in the SPD group with a $19.5 \%$ protein proportion $(p<0.001$ for protein intake percentage, Table 3 ). After the mean treatment duration of 1.2 years in obesity MDT in PKUFH, weight loss of $9 \mathrm{~kg}$ was observed in both groups. According to the 
TABLE 2: Variation of anthropometric and metabolic indicators after treatment of obesity MDT.

\begin{tabular}{|c|c|c|c|c|}
\hline & $n$ & Baseline & After follow-up & $p$ value* \\
\hline Weight (kg) & 119 & $89.7 \pm 20.0$ & $80.9 \pm 17.1$ & $<0.001$ \\
\hline Lean body mass (kg) & 119 & $51.7 \pm 10.9$ & $50.5 \pm 10.0$ & $<0.001$ \\
\hline Hip circumference $(\mathrm{cm})$ & 119 & $109.9 \pm 10.8$ & $105.8 \pm 9.5$ & $<0.001$ \\
\hline Muscle mass of lower extremities (kg) & 119 & $16.1 \pm 3.6$ & $15.7 \pm 3.4$ & $<0.001$ \\
\hline Body adiposity index & 119 & $33.9 \pm 4.9$ & $32.0 \pm 4.3$ & $<0.001$ \\
\hline Fat-free mass index & 119 & $17.0 \pm 6.2$ & $15.2 \pm 7.2$ & 0.043 \\
\hline Fasting plasma glucose $(\mathrm{mmol} / \mathrm{L})$ & 119 & $6.0 \pm 1.5$ & $5.5 \pm 0.9$ & 0.002 \\
\hline Fasting insulin $(\mu \mathrm{IU} / \mathrm{mL})$ & 119 & $19.0 \pm 10.8$ & $13.8 \pm 6.6$ & 0.002 \\
\hline HOMA-IR & 119 & $4.8 \pm 2.6$ & $3.2 \pm 1.5$ & $<0.001$ \\
\hline НОМА- $\beta$ & 119 & $256.7 \pm 39.1$ & $202.9 \pm 21.4$ & 0.134 \\
\hline HbAlc (\%) & 34 & $6.89 \pm 0.26$ & $6.33 \pm 0.93$ & 0.038 \\
\hline $\operatorname{ALT}(\mathrm{U} / \mathrm{L})$ & 54 & $36.0 \pm 3.3$ & $24.7 \pm 2.2$ & 0.001 \\
\hline AST (U/L) & 53 & $23.8 \pm 1.6$ & $20.1 \pm 1.0$ & 0.021 \\
\hline Uric acid $(\mu \mathrm{mol} / \mathrm{L})$ & 53 & $359.0 \pm 91.9$ & $351.6 \pm 74.6$ & 0.480 \\
\hline Triglyceride $(\mathrm{mmol} / \mathrm{L})$ & 54 & $2.15 \pm 0.23$ & $2.06 \pm 0.37$ & 0.745 \\
\hline TCHO (mmol/L) & 54 & $4.70 \pm 0.14$ & $4.86 \pm 0.17$ & 0.320 \\
\hline HDL-c (mmol/L) & 54 & $1.06 \pm 0.26$ & $1.19 \pm 0.30$ & $<0.001$ \\
\hline LDL-c (mmol/L) & 54 & $2.86 \pm 0.96$ & $2.85 \pm 0.88$ & 0.963 \\
\hline
\end{tabular}

ALT: glutamic-pyruvic transaminase; AST: glutamic-oxaloacetic transaminase; TCHO: total cholesterol; HDL-c: high-density lipoprotein cholesterol; LDL-c: low-density lipoprotein cholesterol. ${ }^{*} \chi^{2}$ test for categorical variables and Student's $t$-test for continuous variables.

guidelines on obesity, weight loss of $5 \%$ to $10 \%$ is the first goal of lifestyle intervention treatment [1]. Defining body weight reduction over $5 \%$ of the baseline value as successful weight loss, the success rate was $77.8 \%$ in the HPD group and $68.5 \%$ in the SPD group $(p=0.351)$. Considering the reduction of body weight over $10 \%$ as successful weight loss, the rate of success was $48.1 \%$ in the HPD group and $34.8 \%$ in the SPD group $(p=0.208)$. The reduction of visceral fat area, maintenance of lean body mass, body adiposity index, and fat-free mass index showed no significance between the HPD and SPD groups, as well as glucose metabolic variables including HOMA-IR and HOMA- $\beta$ (Table 3).

\section{Discussion}

This study provides insight into the effectiveness of applying a multidisciplinary team for weight-loss intervention in the real world. In order to explore the effectiveness of a groupbased weight-loss clinic, body weight was assessed during the follow-up. Overall, our results revealed that body weight significantly decreased across the meantime span of 1.2 years of MDT treatment. It also demonstrated that a multidisciplinary weight-loss intervention could be more efficient in improving weight control and metabolic variables than traditional methods.

The obesity mechanism is complex. Besides genetic and environmental factors, the involvement of the opioid and endorphinergic systems has a critical role in feeding behavior, which brings new insight for future obesity medication [13]. In addition, cytokines, including adiponectin, contribute to the pathogenesis of insulin resistance in individuals with normal glucose tolerance and the development of obesity [14].

Over recent years, the lack of knowledge and awareness of obesity has become the main barrier to weight control, especially in China. Other barriers include a lack of motivation and previous negative experience about lifestyle intervention [15]. Our multidisciplinary team for obesity in Peking University First Hospital provides regular obesity education programs for obese patients, where peer support activities are regularly held since the first visit. Previous randomized controlled trials indicated that behavioral intervention could improve weight loss $[16,17]$. Among the patients in our study, $68.1 \%$ received only education programs, a calorie-reduced meal plan, and an exercise training guide. A significant weight reduction was achieved without pharmacotherapy or bariatric surgery. In Gong et al.'s diabetes prevention study in China, body weight dropped over the 23-year follow-up with lifestyle intervention [18]. The participants in our study achieved the mean weight loss of $8.8 \mathrm{~kg}$, including $1.2 \mathrm{~kg}$ loss of lean body mass, and $0.4 \mathrm{~kg}$ loss of lower extremity muscle mass that was maintained through the whole follow-up duration. Medication for weight reduction and glycemic control, including metformin, orlistat, and liraglutide, was prescribed to $31.9 \%$ of the patients in our study. A prospective study proved that metformin could reduce weight and cardiovascular risk by reducing endothelial dysfunction [19].

Body adiposity index (BAI), which is calculated from the hip circumference and height, is considered as a reliable scale that can directly estimate the percentage of adiposity, 
TABLE 3: Comparison of diet, anthropometric and biochemical variables between SPD and HPD group after treatment of obesity MDT.

\begin{tabular}{|c|c|c|c|}
\hline & HPD group $(n=27)$ & SPD group $(n=92)$ & $p$ value* \\
\hline Calorie intake during diet $(\mathrm{kcal} / \mathrm{d})$ & $1389.0 \pm 440.9$ & $1393.0 \pm 431.5$ & 0.966 \\
\hline Protein intake proportion during diet (\%) & $29.9 \pm 6.3$ & $19.5 \pm 3.6$ & $<0.001$ \\
\hline Weight after follow-up (kg) & $80.4 \pm 17.7$ & $80.8 \pm 17.2$ & 0.917 \\
\hline Weight reduction $(\mathrm{kg})$ & $9.0 \pm 1.5$ & $9.0 \pm 1.1$ & 0.992 \\
\hline Percentage of weight loss (\%) & $9.8 \pm 1.5$ & $9.3 \pm 0.9$ & 0.780 \\
\hline Weight loss > 5\% $(n, \%)$ & $21,77.8 \%$ & $63,68.5 \%$ & 0.351 \\
\hline Weight loss $>10 \%(n, \%)$ & $13,48.1 \%$ & $32,34.8 \%$ & 0.208 \\
\hline Hip circumference $(\mathrm{cm})$ & $105.1 \pm 10.3$ & $106.0 \pm 9.4$ & 0.684 \\
\hline Visceral fat area $\left(\mathrm{cm}^{2}\right)$ & $131.8 \pm 54.7$ & $134.7 \pm 43.6$ & 0.797 \\
\hline Lean body mass (kg) & $49.5 \pm 9.8$ & $50.2 \pm 9.9$ & 0.744 \\
\hline Muscle mass of lower extremities (kg) & $15.3 \pm 3.4$ & $15.6 \pm 3.4$ & 0.737 \\
\hline Body adiposity index & $31.7 \pm 4.0$ & $32.2 \pm 4.3$ & 0.652 \\
\hline Fat-free mass index & $14.9 \pm 7.4$ & $14.9 \pm 7.4$ & 0.965 \\
\hline Fasting plasma glucose $(\mathrm{mmol} / \mathrm{L})$ & $5.5 \pm 1.0$ & $5.6 \pm 0.9$ & 0.726 \\
\hline Fasting insulin (uIU/mL) & $12.8 \pm 7.9$ & $14.0 \pm 6.2$ & 0.615 \\
\hline НОМА- $\beta$ & $71.9 \pm 25.5$ & $54.0 \pm 11.6$ & 0.485 \\
\hline HOMA-IR & $1.0 \pm 0.3$ & $1.0 \pm 0.2$ & 0.882 \\
\hline
\end{tabular}

${ }^{*} \chi^{2}$ test for categorical variables and Student's $t$-test for continuous variables.

with higher sensitivity and specificity than traditional anthropometric parameters [20]. A previous study suggested that a lean body mass, especially the muscle mass of lower extremities, was positively associated with a longer life span [21]. During weight loss treatment, both adipose tissue and lean body mass were reduced over time. Our aim was to lower adipose mass and simultaneously keep lean body mass through a high-protein diet and resistance exercise training. Our results revealed a subtle loss of lean body mass, fat-free mass index (FFMI), and lower extremity muscle mass accompanied by a significant reduction of BAI and body weight.

At the initial visit, anthropometric data and metabolic parameters of the patients were collected. Among 119 patients (26 to 52 years old), $38.8 \%$ of patients suffered from hyperlipidemia, $39.5 \%$ from type 2 diabetes, $37.4 \%$ from hypertension, and $60.4 \%$ from NAFLD. Previous data suggested that obesity and high-fat meal could result in the increase of tumor necrosis factor $\alpha$ (TNF- $\alpha$ ) and endothelial dysfunction [22], which may lead to the deterioration of metabolic indicators. Also, patients with diabetes have a higher risk of atherosclerosis progression, which might be related to the ubiquitin-proteasome system [23]. In our study, metabolic indicators, including $\mathrm{HDL}-\mathrm{c}$, transaminase, FPG, fasting insulin, and HOMA-IR, were improved along with weight loss. The reversing effect of insulin resistance in the participants indicated the necessity of regular screening for diabetes in obese patients with chronic status of insulin resistance and the urgency of weight loss.

A previous study has proven that common mechanisms, including endoplasmic reticulum stress, contribute to NAFLD and obesity [24]. In the present study, the occur- rence of NAFLD in our study population reached $60.4 \%$, while the prevalence of NAFLD in the world population was around $16 \%$ [25]. Along with the elevating body weight and BMI, the prevalence of nonalcoholic steatohepatitis (NASH), which is characterized by increased transaminase, could be further lifted. Once the transaminase is above the upper limit, a possibility of cirrhosis reaches 5-8\% in 5 years of follow-up [26]. For both NASH and NAFLD, aside from lipid-lowering agents, one of the key management approaches was weight reduction [27]. Corroborating our findings, transaminase could be reduced after moderate weight loss and was beneficial for the management of NASH and NAFLD, as well as the prevention of cirrhosis.

Subgroup analysis of the high-protein diet and standard calorie-restricted diet was performed in our study to investigate the weight loss efficiency and metabolic alternation of the two diets. Previous studies, including some small scale randomized controlled studies (RCT), reported controversial conclusions about HPD. High-protein content in a diet resulted in more satiety, shrinking energy intake, and greater dietary thermogenesis. In an RCT in patients with metabolic syndrome, no significant differences in weight loss and biomarkers of metabolic syndrome were examined. Still, the participants with a higher adherence rate in the HPD group lost significantly more weight than those in the SPD group [28]. Patients in our study were recommended high-protein meal plans if they had a normal renal function at the first visit and were assigned to the HPD or SPD group in accordance with the diet questionnaire at each visit when daily protein intake was above $20 \%$ of the total calorie intake of the HPD group. Therefore, the number of participants in the HPD group was relatively small and weakened the statistical 
significance of weight reduction and glucose metabolic variables when comparing the two groups. The rate of weight loss over $5 \%$ was $77.8 \%$ in the HPD group and $68.5 \%$ in the SPD group ( $p=0.351)$, and the rate of losing weight over $10 \%$ was $48.1 \%$ in the HPD group and $34.8 \%$ in the SPD group $(p=0.208)$. There was a tendency, but there was no statistical difference in weight loss efficiency in the HPD group; hence, no predominance of metabolic indicators in HPD was discovered in our study. A larger population and further study of the obesity MDT in PKUFH could elaborate more on the possible advantage of HPD.

This study has a few limitations. First, the data and results were collected from real-world cohort data, and it was impossible to avoid confounding factors that might affect the reliability of the conclusion. During our study, patients received a multidisciplinary treatment. For example, it was common that a patient underwent lifestyle intervention and acupuncture for a couple of months and was put on metformin for a year and then finally decided to have a bariatric surgery. Herein, the proportion of each kind of treatment was recorded in our study, and it was difficult to perform statistical analysis for the effectiveness of different ways for weight reduction. The follow-up duration and sample size were relatively small in our study. Continuous follow-up and patient enrollment, as well as a longer duration of follow-up and enlarged sample size, may further corroborate our results.

\section{Conclusions}

A multidisciplinary team for obesity management could significantly reduce body weight and improve metabolic indicators, including HDL-c, transaminase, and insulin resistance. A high-protein diet does not produce better weight control or body composition compared with a standard calorie-restricted diet.

\section{Data Availability}

Data is available at any time after contacting the corresponding author via email.

\section{Ethical Approval}

The study protocol followed the guidelines for human studies and was conducted ethically in accordance with the World Medical Association Declaration of Helsinki. Ethical approval was given from the Ethics Committee of Peking University First Hospital.

\section{Conflicts of Interest}

The authors have no conflict of interests.

\section{Authors' Contributions}

$\mathrm{Lu} \mathrm{D}$ conducted data collection and statistical analysis and wrote the manuscript. Yuan $\mathrm{Z}$ designed the research and revised the manuscript. Yang L, Jing L, Wang R, Wang Y, Jiang Y, and Li M participated in patient enrollment and treatment. Zhang J and Guo X revised the manuscript.

\section{Acknowledgments}

The Scientific Research Seed Fund of Peking University First Hospital (2018SF028) supported this study.

\section{References}

[1] M. M. Finucane, G. A. Stevens, M. J. Cowan et al., "National, regional, and global trends in body-mass index since 1980: systematic analysis of health examination surveys and epidemiological studies with 960 country-years and 9.1 million participants," The Lancet, vol. 377, no. 9765, pp. 557-567, 2011.

[2] W. T. Garvey, "New tools for weight-loss therapy enable a more robust medical model for obesity treatment: rationale for a complications-centric approach," Endocrine Practice, vol. 19, no. 5, pp. 864-874, 2013.

[3] R.-Q. Zhang, J. Tan, F.-Y. Li, Y.-H. Ma, L.-X. Han, and X.L. Yang, "Acupuncture for the treatment of obesity in adults: a systematic review and meta-analysis," Postgraduate Medical Journal, vol. 93, no. 1106, pp. 743-751, 2017.

[4] A. V. Ries, L. T. Blackman, R. A. Page et al., "Goal setting for health behavior change: evidence from an obesity intervention for rural low-income woman," Rural and Remote Health, vol. 14, p. 2682, 2014.

[5] W. T. Garvey, J. I. Mechanick, E. M. Brett et al., “American Association of Clinical Endocrinologists and American College of Endocrinology Comprehensive Clinical Practice Guidelines Formedical Care of Patients with Obesity," Endocrine Practice, vol. 22, pp. 1-203, 2016.

[6] C. M. Apovian, W. T. Garvey, and D. H. Ryan, "Challenging obesity: patient, provider, and expert perspectives on the roles of available and emerging nonsurgical therapies," Obesity, vol. 23, Suppl 2, pp. S1-S26, 2015.

[7] F. Gillison, A. Stathi, P. Reddy et al., "Processes of behavior change and weight loss in a theory-based weight loss intervention program: a test of the process model for lifestyle behavior change," International Journal of Behavioral Nutrition and Physical Activity, vol. 12, no. 1, p. 2, 2015.

[8] C. Maiz, J. Alvarado, N. Quezada, J. Salinas, R. Funke, and C. Boza, "Bariatric surgery in 1119 patients with preoperative body mass index $<35\left(\mathrm{~kg} / \mathrm{m}^{2}\right)$ : results at 1 year," Surgery for Obesity and Related Diseases, vol. 11, no. 5, pp. 1127-1132, 2015.

[9] A. Due, S. Toubro, A. R. Skov, and A. Astrup, "Effect of normal-fat diets, either medium or high in protein, on body weight in overweight subjects: a randomised 1-year trial," International Journal of Obesity and Related Metabolic Disorders, vol. 28, no. 10, pp. 1283-1290, 2004.

[10] R. N. Bergman, D. Stefanovski, T. A. Buchanan et al., "A better index of body adiposity," Obesity, vol. 19, no. 5, pp. 1083-1089, 2011.

[11] T. B. VanItallie, M. U. Yang, S. B. Heymsfield, R. C. Funk, and R. A. Boileau, "Height-normalized indices of the body's fatfree mass and fat mass: potentially useful indicators of nutritional status," The American Journal of Clinical Nutrition, vol. 52, no. 6, pp. 953-959, 1990.

[12] D. R. Matthews, J. P. Hosker, A. S. Rudenski, B. A. Naylor, D. F. Treacher, and R. C. Turner, "Homeostasis model assessment: insulin resistance and beta-cell function from fasting plasma glucose and insulin concentrations in man," Diabetologia, vol. 28, no. 7, pp. 412-419, 1985. 
[13] D. Cozzolino, G. Sessa, T. Salvatore et al., "The involvement of the opioid system in human obesity: a study in normal weight relatives of obese people," The Journal of Clinical Endocrinology and Metabolism, vol. 81, no. 2, pp. 713-718, 1996.

[14] F. C. Sasso, P. C. Pafundi, R. Marfella et al., "Adiponectin and insulin resistance are related to restenosis and overall new PCI in subjects with normal glucose tolerance: the prospective AIRE study," Cardiovascular Diabetology, vol. 18, no. 1, p. 24, 2019.

[15] A. A. H. Schalkwijk, G. Nijpels, S. D. M. Bot, and P. J. M. Elders, "Health care providers' perceived barriers to and need for the implementation of a national integrated health care standard on childhood obesity in the Netherlands - a mixed methods approach," BMC Health Services Research, vol. 16, no. 1, 2016.

[16] A. A. Gorin, H. A. Raynor, J. Fava et al., "Randomized controlled trial of a comprehensive home environment-focused weight-loss program for adults," Health Psychology, vol. 32, no. 2, pp. 128-137, 2013.

[17] I. Shai, D. Schwarzfuchs, Y. Henkin et al., "Weight loss with a low-carbohydrate, Mediterranean, or low-fat diet," The New England Journal of Medicine, vol. 359, no. 3, pp. 229-241, 2008.

[18] Q. Gong, P. Zhang, J. Wang et al., "Changes in mortality in people with IGT before and after the onset of diabetes during the 23-year follow-up of the Da Qing Diabetes Prevention Study," Diabetes Care, vol. 39, no. 9, pp. 1550-1555, 2016.

[19] C. Sardu, P. Paolisso, C. Sacra et al., "Effects of metformin therapy on coronary endothelial dysfunction in patients with prediabetes with stable angina and nonobstructive coronary artery stenosis: the CODYCE multicenter prospective study," Diabetes Care, vol. 42, no. 10, pp. 1946-1955, 2019.

[20] A. Geliebter, D. Atalayer, L. Flancbaum, and C. D. Gibson, "Comparison of body adiposity index (BAI) and BMI with estimations of \% body fat in clinically severe obese women," Obesity, vol. 21, no. 3, pp. 493-498, 2013.

[21] A. Due, T. M. Larsen, H. Mu, K. Hermansen, S. Stender, and A. Astrup, "Comparison of 3 ad libitum diets for weight-loss maintenance, risk of cardiovascular disease, and diabetes: a 6-mo randomized controlled trial," The American Journal of Clinical Nutrition, vol. 88, no. 5, pp. 1232-1241, 2008.

[22] K. Esposito, M. Ciotola, F. C. Sasso et al., "Effect of a single high-fat meal on endothelial function in patients with the metabolic syndrome: role of tumor necrosis factor- $\alpha$," Nutrition, Metabolism, and Cardiovascular Diseases, vol. 17, no. 4, pp. 274-279, 2007.

[23] R. Marfella, M. D'Amico, C. Di Filippo et al., "The possible role of the ubiquitin proteasome system in the development of atherosclerosis in diabetes," Cardiovascular Diabetology, vol. 6, no. 1, p. $35,2007$.

[24] M. J. Pagliassotti, P. Y. Kim, A. L. Estrada, C. M. Stewart, and C. L. Gentile, "Endoplasmic reticulum stress in obesity and obesity-related disorders: an expanded view," Metabolism, vol. 65, no. 9, pp. 1238-1246, 2016.

[25] S. Milic, D. Lulic, and D. Stimac, "Non-alcoholic fatty liver disease and obesity: biochemical, metabolic and clinical presentations," World Journal of Gastroenterology, vol. 20, no. 28, pp. 9330-9337, 2014.

[26] L. A. Streba, C. C. Vere, I. Rogoveanu, and C. T. Streba, "Nonalcoholic fatty liver disease, metabolic risk factors, and hepatocellular carcinoma: an open question," World Journal of Gastroenterology, vol. 21, no. 14, pp. 4103-4110, 2015.
[27] S. Liyanagedera, R. P. Williams, S. Veraldi, V. Nobili, and J. P. Mann, "The pharmacological management of NAFLD in children and adolescents," Expert Review of Clinical Pharmacology, vol. 10, no. 11, pp. 1225-1237, 2017.

[28] I. Campos-Nonato, L. Hernandez, and S. Barquera, "Effect of a high-protein diet versus standard-protein diet on weight loss and biomarkers of metabolic syndrome: a randomized clinical trial," Obesity Facts, vol. 10, no. 3, pp. 238-251, 2017. 